University of Nebraska - Lincoln

DigitalCommons@University of Nebraska - Lincoln

Faculty Publications from the Department of Electrical \& Computer Engineering, Department Electrical and Computer Engineering

2-12-2001

\title{
Infrared response of multiple-component free-carrier plasma in heavily doped $p$-type GaAs
}

\author{
S. Zangooie \\ University of Nebraska-Lincoln \\ Mathias Schubert \\ University of Nebraska-Lincoln, mschubert4@unl.edu \\ Daniel W. Thompson \\ University of Nebraska-Lincoln, dthompson2@unl.edu \\ John A. Woollam \\ University of Nebraska-Lincoln, jwoollam1@unl.edu
}

Follow this and additional works at: https://digitalcommons.unl.edu/electricalengineeringfacpub

Part of the Electrical and Computer Engineering Commons

Zangooie, S.; Schubert, Mathias; Thompson, Daniel W.; and Woollam, John A., "Infrared response of multiple-component free-carrier plasma in heavily doped p-type GaAs" (2001). Faculty Publications from the Department of Electrical and Computer Engineering. 3.

https://digitalcommons.unl.edu/electricalengineeringfacpub/3

This Article is brought to you for free and open access by the Electrical \& Computer Engineering, Department of at DigitalCommons@University of Nebraska - Lincoln. It has been accepted for inclusion in Faculty Publications from the Department of Electrical and Computer Engineering by an authorized administrator of DigitalCommons@University of Nebraska - Lincoln. 


\title{
Infrared response of multiple-component free-carrier plasma in heavily doped $p$-type GaAs
}

\author{
S. Zangooie, M. Schubert, ${ }^{\text {a) }}$ D. W. Thompson, and J. A. Woollam ${ }^{\text {b) }}$ \\ Center for Microelectronic and Optical Materials Research and Department of Electrical Engineering, \\ University of Nebraska, Lincoln, Nebraska 68588-0511
}

(Received 24 August 2000; accepted for publication 20 November 2000)

\begin{abstract}
Spectroscopic ellipsometry is used to measure the dielectric function of heavily doped $p$-type GaAs for wave numbers from 100 to $2000 \mathrm{~cm}^{-1}$. Due to partial filling of the heavy- and light-hole valence bands, heavy holes as well as light holes form a multiple-component plasma coupled with longitudinal optical phonons. Line-shape analysis of the infrared response allows differentiating between light- and heavy-hole contributions to the carrier plasma, and the results observed suggest nonparabolicity effects of the heavy- and light-hole valence bands in GaAs. (C) 2001 American Institute of Physics. [DOI: 10.1063/1.1343490]
\end{abstract}

Infrared optical properties of semiconductors are influenced significantly by the free-carrier response. ${ }^{1,2}$ Optical characteristics of $p$-type GaAs is complicated by penetration of the Fermi level into the valence bands at the $\Gamma$ point of the Brillouin zone, providing contributions from heavy holes (hhs) as well as light holes (lhs) to the free-carrier plasma. ${ }^{3,4}$ In addition, an accurate characterization of GaAs surfaces requires consideration of further complexities such as a depleted surface layer caused by Fermi-level pinning to midband-gap surface states. ${ }^{4,5}$ Infrared spectroscopic ellipsometry (IRSE) is nondestructive and intrinsically sensitive to thin-film optical properties. IRSE has been employed previously for characterization of carrier-depleted surface layers in $n$-type GaAs (Ref. 5) and $n$-type GaN. ${ }^{6}$ Electrical properties of doped semiconductors are traditionally measured using the electrical Hall effect. However, complexities normally accompanied with electrical Hall measurements of $p$-type GaAs, such as surface contamination due to electrical contacts, or non-Ohmic contact behavior, ${ }^{4}$ motivates employment of additional nondestructive and accurate characterization techniques. In this work, IRSE is used to simultaneously determine the real and imaginary parts of the dielectric function of heavily doped $p$-type GaAs. It is shown that IRSE can be used to differentiate between contributions from different carrier types in multiple-component plasma interactions, i.e., between hh and $\mathrm{lh}$ plasma components as well as between their contributions to the dielectric function. Here, carrier concentration and mobility parameters are of particular interest. Accurate knowledge of these parameters is crucial in design of device structures that utilize heavily doped $p$-type GaAs. For data analysis it is assumed that the bands are parabolic, i.e., the effective masses are assumed to be the same as those from the top of the valence bands at the $\Gamma$ point of the Brillouin zone, and do not depend on the

a) Present address: Universität Leipzig, Fakultät für Physik und Geowissenschaften, Arbeitsgruppe Festkörperoptik, Linnéstrasse 5, Leipzig 04103, Germany.

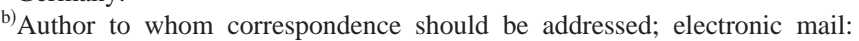
jwoollam@unl.edu
Fermi-level energy. The results obtained here, however, suggest nonparabolicity effects of the valence bands.

IRSE measurements at room temperature were carried out for wave numbers from 500 to $2000 \mathrm{~cm}^{-1}$, with $2 \mathrm{~cm}^{-1}$ resolution, using a commercially available Fouriertransform-based spectroscopic ellipsometer at incidence angles of $50^{\circ}$ and $70^{\circ}$. Data in the $100-500 \mathrm{~cm}^{-1}$ spectral region (far-IR) were acquired using a prototype IRSE equipped with a He-cooled bolometer detector unit. The far-IR measurements are necessary for accurate determination of the GaAs phonon-mode frequencies and the freecarrier parameters. Zn-doped $p$-type GaAs wafers with (100) crystal orientation were obtained from the Materials Technology International (MTI) Corporation. The carrier concentration and carrier mobility, reported by the manufacturer, are $1.4 \times 10^{19} \mathrm{~cm}^{-3}$ and $70 \mathrm{~cm}^{2} \mathrm{~V}^{-1} \mathrm{~s}^{-1}$, respectively.

The measured quantity in ellipsometry ${ }^{7}$ is the so-called complex reflectance ratio, which is expressed as

$$
\rho=\frac{R_{p}}{R_{S}}=\tan (\psi) \exp (i \Delta)
$$

$R_{p}$ and $R_{s}$ are the complex reflection coefficients for $p$ - and $s$-polarized light, respectively. The amplitude ratio $\tan (\psi)$ and the relative difference in phase change $\Delta$ for the $p$ - and $s$-polarized light components upon reflection from the sample surface are the standard ellipsometry parameters. From experimentally determined $\rho$, information can be obtained about the optical properties of the material under study in terms of the complex dielectric function $\varepsilon=\varepsilon_{1}$ $+i \varepsilon_{2}$. Optical properties of the $p$-type GaAs wafers in terms of the $\varepsilon$ are modeled using the following expression: ${ }^{8}$

$\varepsilon(\omega)=\varepsilon_{\infty}+\frac{\varepsilon_{\infty}\left(\omega_{\mathrm{LO}}^{2}-\omega_{\mathrm{TO}}^{2}\right)}{\omega_{\mathrm{TO}}^{2}-\omega^{2}-i \omega \gamma}-\frac{\varepsilon_{\infty} \omega_{p h h}^{2}}{\omega\left(\omega+i \Gamma_{\mathrm{hh}}\right)}-\frac{\varepsilon_{\infty} \omega_{p \mathrm{hh}}^{2}}{\omega\left(\omega+i \Gamma_{\mathrm{lh}}\right)}$.

$\varepsilon_{\infty}$ and $\gamma$ are the high-frequency dielectric function and the transverse-optical (TO) phonon-damping parameters, respectively. $\omega_{\mathrm{TO}}$ and $\omega_{\mathrm{LO}}$ represent the IR-active TO and longitudinal-optical (LO) phonon frequencies, respectively. The third and fourth terms in Eq. (2) are the Drude expressions, which are introduced to consider effects of the 
multiple-component free-carrier plasma, which consists of heavy hole and light hole components in the highly doped semiconductor. The screened plasmon frequencies $\left(\omega_{p, \mathrm{lh}, \mathrm{hh}}\right)$ and the plasmon broadening parameters $\left(\Gamma_{\mathrm{lh}, \mathrm{hh}}\right)$ are related to the carrier concentrations $\left(p_{\mathrm{lh}, \mathrm{hh}}\right)$ and mobilities $\left(\mu_{\mathrm{lh}, \mathrm{hh}}\right)$ according to the following relations:

$$
\begin{aligned}
& \omega_{p j}=\sqrt{\frac{p_{j} e^{2}}{\varepsilon_{0} \varepsilon_{\infty} m_{j}^{*}}}, \\
& \Gamma_{j}=\frac{e}{m^{*} \mu_{j}}, \quad j=\mathrm{lh}, \mathrm{hh} .
\end{aligned}
$$

$\varepsilon_{0}, e$, and $m_{j}^{*}$ are the vacuum permitivity, electrical unity charge, and the effective mass of the free carriers, respectively. IRSE is only sensitive to $\left(p / m^{*}\right)$ and $1 /\left(\mu m^{*}\right)$ because these ratios parametrize the dielectric response of a multiple-component carrier plasma. Thus, to calculate the concentration and mobility of the carriers from $\varepsilon$, the parameters $m_{j}^{*}$ must be known. For the calculations here the averaged $\mathrm{hh}$ effective mass of $0.56 \mathrm{~m}_{0}$, and the averaged $\mathrm{lh}$ effective mass of $0.08 m_{0}$ are used $\left(m_{0}\right.$ is the free-electron mass). ${ }^{3,4}$ Note that one could not differentiate between $p_{\mathrm{lh}}$ and $p_{\mathrm{hh}}\left(\right.$ or $\omega_{p \mathrm{lh}}$ and $\omega_{p \mathrm{hh}}$ ) if both carrier species would have the same broadening parameters given by the mass-mobility product. It is the different strength of absorption of both types that allows us to separate their different contributions to $\varepsilon$. Furthermore, depending on the choice of $m_{j}^{*}$, the Drude expressions in Eq. (2) provide two different sets of solutions for each species. Here, the solution with larger carrier concentration is assumed to represent the heavy holes [see, e.g., Eq. (6)]. Note that in Zn-doped GaAs the ionizedimpurity scattering was found as the most significant scattering mechanism. ${ }^{4}$ We further note that Kukharskii's ${ }^{10}$ factorized form of $\varepsilon$, which allows for independent broadening of $\mathrm{TO}$ and LO branches in polar materials, did not improve the fits to the experimental data and was, therefore, abandoned. This model was used by Kukharskii to explain IR-reflectivity spectra of heavily doped $n$-type GaAs. ${ }^{10}$

The difference in the free-carrier concentration between the depleted layer and the underlying bulk material yields static electric-field gradients influencing the long-range Coulomb forces. Hence, different LO phonon frequencies are encountered in the depleted layer and the underlying heavily doped GaAs. The phenomenon results in a characteristic signature within the IRSE data above the TO phonon frequency and near to the LO frequency of intrinsic GaAs. The signature, which is distinguished as a minimum in $\psi$ spectra, can be utilized for determination of the thickness $d$ of the depleted layer. The phenomenon was first observed by Berreman in thin LiF films on silver. ${ }^{11}$ The Berreman effect is also reported for $\mathrm{SiC}$ homostructures consisting of low-doped epitaxial layers on highly doped substrates. ${ }^{5,12}$ It is often assumed that at a distance (equal to $d$ ) away from the surface the carrier concentration $p$ abruptly rises to the bulk value, which yields for the depletion depth $d=\sqrt{\left(2 \underline{\varepsilon}_{s} \varepsilon_{0} \phi_{0} / p / e\right)}$ where $\varepsilon_{s}=12.5$ is the static dielectric constant for GaAs, and $e \phi_{0}=0.5 \mathrm{eV}$ is the electrostatic potential energy. ${ }^{4,8}$ For carrier concentrations $10^{18}$ and $10^{19} \mathrm{~cm}^{-3}, d$ equals 26.3 and 8.3 $\mathrm{nm}$, respectively. Note, however, that Eq. (5) overestimates the depletion layer depth. ${ }^{4}$ The large wavelength near

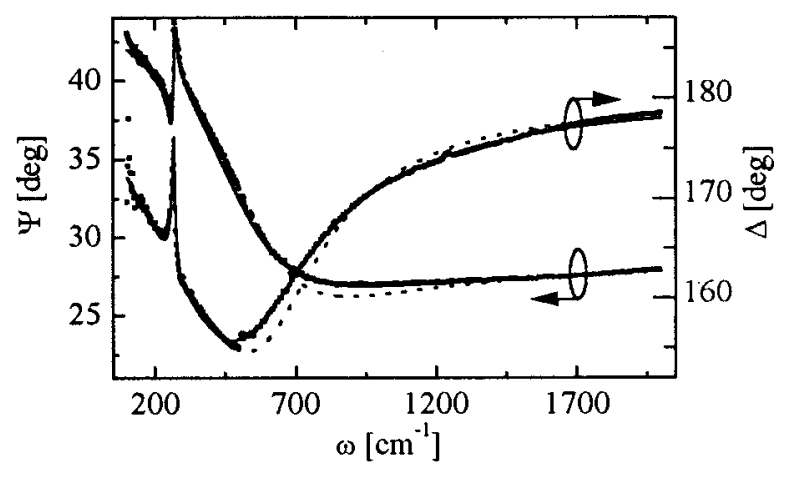

FIG. 1. Experimental (solid circles) and calculated [solid lines; after Eq. (7)] $\psi$ and $\Delta$ spectra of the heavily doped $p$-type GaAs sample at a $50^{\circ}$ angle of incidence. The dotted lines represent the best-fit model calculation assuming $\left(p_{\mathrm{hh}} / p_{\mathrm{lh}}\right)^{2}=\left(m_{\mathrm{hh}} / m_{\mathrm{lh}}\right)^{3}$. The derivative-like structure in $\psi$ and $\Delta$ at $\omega$ $\sim 267 \mathrm{~cm}^{-1}$ is due to resonant excitation of the GaAs TO mode.

$\omega_{\mathrm{LO}}(\lambda \sim 26 \mu \mathrm{m})$, and negligible changes of the polarization state of the reflected light by the very thin depletion layer in the sample studied here, explain lack of a clear depletionlayer-related signature in our IRSE data. This layer is, therefore, not considered in the analysis.

Figure 1 presents experimental and calculated IRSE spectra in terms of $\psi$ and $\Delta$ for the heavily doped $p$-type GaAs wafer. For clarity, data are shown for the $50^{\circ}$ angle of incidence only. Note the excellent agreement between measured and calculated spectra. Experimental data above the TO phonon frequency and near the position of the plasma minimum $\left[\varepsilon_{1}\left(\omega \sim 470 \mathrm{~cm}^{-1}\right) \sim 1\right.$; see, also, Fig. 2] provide sensitivity to the free-carrier parameters. The best-fit calculation parameter values and the corresponding confidence intervals are given in Table I. Values of $\varepsilon_{\infty}, \omega_{\mathrm{TO}}, \omega_{\mathrm{LO}}$, and $\gamma$ for heavily doped GaAs were also reported in Ref. 4, and compare well to those found here (Table I). Moreover, the
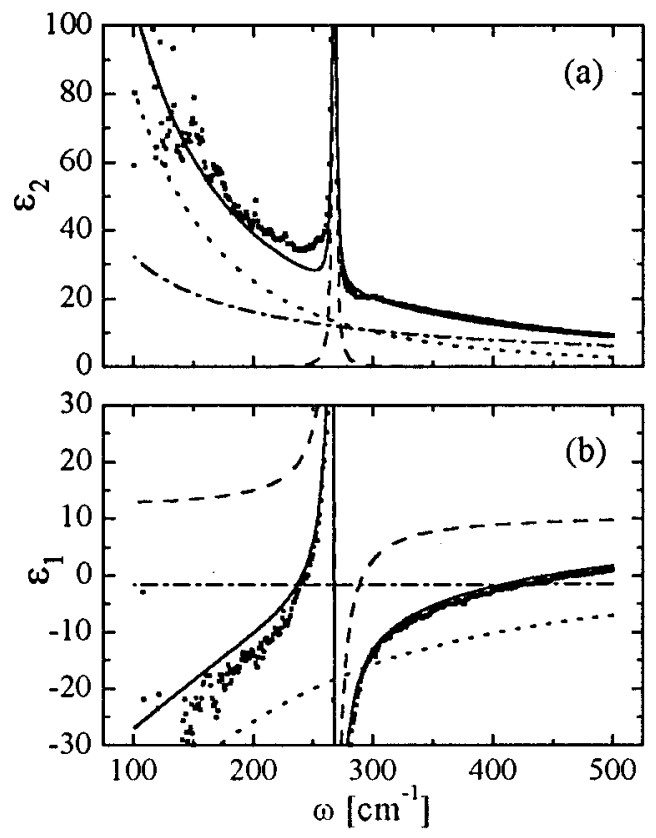

FIG. 2. Real (a) and imaginary part (b) of the dielectric function of GaAs with a multiple-component plasma using the parameter values given in Table I (solid lines), and with the lattice contribution considered only (dashed lines). Separate contributions from heavy (dotted lines) and light holes (dash-dotted lines) to $\varepsilon_{1}$ and $\varepsilon_{2}$ are also shown. 
TABLE I. Best-fit parameters including $90 \%$ confidence intervals. $m_{\mathrm{hh}}$ $=0.56 m_{0}$ and $m_{\mathrm{lh}}=0.08 m_{0}$ are used during IRSE data analysis. Parameters obtained by Raman scattering in Ref. 4, for similar carrier concentrations and mobility, are shown for comparison. The nominal hole concentration and mobility reported by the sample supplier are $1.4 \times 10^{19} \mathrm{~cm}^{-3}$ and 70 $\mathrm{cm}^{2} / \mathrm{V} \mathrm{s}$.

\begin{tabular}{lll}
\hline \hline Model parameters & \multicolumn{1}{c}{ This work } & \multicolumn{1}{c}{ Ref. 4 } \\
\hline$\varepsilon_{\infty}$ & $10.52 \pm 0.02$ & $(10.6)^{\mathrm{a}}$ \\
$\omega_{\mathrm{TO}}\left(\mathrm{cm}^{-1}\right)$ & $267.4 \pm 0.1$ & 268.1 \\
$\omega_{\mathrm{LO}}\left(\mathrm{cm}^{-1}\right)$ & $291.5 \pm 0.2$ & 291.4 \\
$\gamma\left(\mathrm{cm}^{-1}\right)$ & $3.1 \pm 0.1$ & 2 \\
$p_{\mathrm{hh}}\left(\mathrm{cm}^{-3}\right)$ & $(1.26 \pm 0.03) \times 10^{19}$ & $1.52 \times 10^{19}$ \\
$p_{\mathrm{lh}}\left(\mathrm{cm}^{-3}\right)$ & $(5.89 \pm 0.02) \times 10^{18}$ & $8.20 \times 10^{17}$ \\
$p_{\text {tot }}=p_{\mathrm{hh}}+p_{\mathrm{lh}}\left(\mathrm{cm}^{-3}\right)$ & $1.85 \times 10^{19}$ & $1.60 \times 10^{19}$ \\
$\mu_{\mathrm{hh}}\left(\mathrm{cm}^{2} \mathrm{~V}^{-1} \mathrm{~s}^{-1}\right)$ & $85 \pm 1$ & 62 \\
$\mu_{\mathrm{lh}}\left(\mathrm{cm}^{2} \mathrm{~V}^{-1} \mathrm{~s}^{-1}\right)$ & $57 \pm 1$ & 163 \\
$\mu_{\mathrm{eff}}\left(\mathrm{cm}^{2} \mathrm{~V}^{-1} \mathrm{~s}^{-1}\right)$ & 76 & 67 \\
\hline \hline
\end{tabular}

${ }^{\mathrm{a}}$ Reference 14 .

effective hole mobility, assuming decoupled valence bands, 3,9

$$
\mu_{\mathrm{eff}}=\frac{p_{\mathrm{hh}} \mu_{\mathrm{lh}}+p_{\mathrm{lh}} \mu_{\mathrm{lh}}}{p_{\mathrm{hh}}+p_{\mathrm{lh}}},
$$

is $76 \mathrm{~cm}^{2} \mathrm{~V}^{-1} \mathrm{~s}^{-1}$, which is in good agreement with the Hall measurements performed on samples reported in Ref. 4 with similar carrier concentrations, and with $70 \mathrm{~cm}^{2} /(\mathrm{V} \mathrm{s})$ reported by the supplier. For a parabolic band, $p$ can be expressed as ${ }^{9}$

$$
p=2\left(\frac{2 \pi k T m^{*}}{h^{2}}\right)^{3 / 2} N_{\nu} \exp \left(\frac{E_{\nu}-E_{f}}{k T}\right),
$$

where $E_{\nu}, E_{f}, k, h$, and $T$ are energy at the top of the valence band, Fermi-level energy, Boltzmann's and Planck's constants, and temperature, respectively. Note that Eq. (6) is valid when $E_{f}>E_{\nu}+4 k T$. According to Eq. (6), in our sample the position of the Fermi level is above the split-off (so) valence band (spin-orbit splitting parameter $\delta_{\mathrm{SO}}$ $=0.36 \mathrm{eV})$. Consideration of the so contribution to $\varepsilon\left(m_{\mathrm{SO}}\right.$ $=0.165 \mathrm{~m}_{0}$ ) did not improve the fit to the experimental data. A consequence of the parabolic-band assumption is that $p_{\mathrm{hh}}$ and $p_{\mathrm{lh}}$ fulfill the following relation: $:^{3,4}$

$$
\frac{p_{\mathrm{hh}}}{p_{\mathrm{lh}}}=\left(\frac{m_{\mathrm{hh}}}{m_{\mathrm{lh}}}\right)^{3 / 2} \text {. }
$$

Using this constraint during analysis deteriorated the agreement between the best-fit parameter calculation and the experimental data (dotted lines in Fig. 1). Parameters $p_{\mathrm{hh}}$ and $p_{\mathrm{lh}}$ obtained here assuming $m_{\mathrm{hh}}=0.56 m_{0}$ and $m_{\mathrm{lh}}$ $=0.08 m_{0}$ are inconsistent with Eq. (7). Hence, the parabolic-band assumption may not hold for large carrier concentrations. Nonparabolicities of the density-of-states effective masses for the hh, lh, and so valence bands in GaAs were predicted from full $\mathbf{k} \cdot p$ theory by Lowney and Kahn. ${ }^{13}$ A better approach for IRSE data analysis would be to allow for the nonlinear dependencies $m_{\mathrm{hh}}\left(p_{\mathrm{hh}}\right)$ and $m_{\mathrm{lh}}\left(p_{\mathrm{lh}}\right)$, e.g., as predicted by Lowney and Kahn, instead of assuming $m_{j}^{*}$ independent of $p_{j}$. However, because IRSE determines the product $p m_{j}^{*}$, and not $m_{j}^{*}$ and $p_{j}$ independently, the results for $p_{j}$ obtained thereby would still rely on the input functions $m_{j}^{*}\left(p_{j}\right)$. Magneto-optic ellipsometry experiments may present an alternative to obtain more independent parameter information.

Figures 2(a) $\left(\varepsilon_{1}\right)$ and 2(b) $\left(\varepsilon_{2}\right)$ show plasma and lattice contributions to $\varepsilon$, according to Eq. (2) using parameter values in Table I. For wave numbers below the TO phonon mode, coupling of hhs to the LO phonons dominates the IR response, whereas for wave numbers above the TO phonon frequency $\varepsilon$ is dominated by lh contributions. In Fig. 2(b) the light-hole carriers generate most of the IR absorption, whereas the heavy-hole plasma interaction dominates the absorption for short wavelengths. Note that a single-component plasma, employing $p_{\text {tot }}, \mu_{\text {eff }}$ given in Table I, assuming $m^{*}$ $=0.43 \mathrm{~m}_{0},{ }^{4}$ fails to mimic $\varepsilon$ of the highly degenerate p-GaAs wafer measured here. For completeness, Fig. 2 also contains the experimental $\Psi$ and $\Delta$ values converted directly into the so-called pseudodielectric function $\langle\varepsilon\rangle$ (solid circles). ${ }^{14}$ Because no overlayer was considered during data analysis $\langle\varepsilon\rangle$ equals $\varepsilon$, which is sufficiently satisfied here except for increasing data noise at the long-wavelength end.

In conclusion, light holes play a more significant role in the absorption mechanism above the TO phonon frequency, whereas for lower frequencies carriers with heavier effective masses dominate the dielectric function line shape. As a consequence, heavy holes and light holes can be separated by an appropriate IRSE measurement and analysis. For highly doped $p$-type GaAs the IRSE data analysis revealed nonparabolicity effects.

Financial support for this study was provided in part by the Swedish Foundation for International Cooperation in Research and Higher Education (STINT), CMOMR at UNL, and NSF Contract No. DMI-9901510.

${ }^{1}$ T. E. Tiwald, J. A. Woollam, S. Zollner, J. Christiansen, R. B. Gregory, T. Wetteroth, S. R. Wilson, and A. P. Powell, Phys. Rev. B 60, 11464 (1999).

${ }^{2}$ H. Nakano, T. Sakamoto, and K. Taniguchi, J. Appl. Phys. 83, 1384 (1998).

${ }^{3}$ S. Adachi, GaAs and Related Materials: Bulk Semiconducting and Superlattice Properties (World Scientific, Singapore, 1994).

${ }^{4}$ G. Irmer, M. Wenzel, and J. Monecke, Phys. Rev. B 56, 9524 (1997).

${ }^{5}$ J. Humliček, R. Henn, and M. Cardona, Appl. Phys. Lett. 69, 2581 (1996).

${ }^{6}$ A. Kasic, M. Schubert, S. Einfeldt, D. Hommel, and T. E. Tiwald, Phys. Rev. B 62, 7365 (2000).

${ }^{7}$ R. M. A. Azzam and N. M. Bashara, Ellipsometry and Polarized Light (North-Holland, New York, 1987).

${ }^{8}$ P. Y. Yu and M. Cardona, Fundamentals of Semiconductors: Physics and Materials Properties (Springer, Berlin, 1996).

${ }^{9}$ C. M. Wolfe, N. Holonyak, Jr., and G. E. Stillman, Physical Properties of Semiconductors (Prentice-Hall, Englewood Cliffs, NJ, 1989).

${ }^{10}$ A. A. Kukharskii, Solid State Commun. 13, 1761 (1973).

${ }^{11}$ D. W. Berreman, Phys. Rev. 130, 2193 (1963).

${ }^{12}$ S. Zollner, J. P. Carrejo, T. E. Tiwald, and J. A. Woollam, Phys. Status Solidi A 208, R3 (1998).

${ }^{13}$ J. R. Lowney and A. H. Kahn, J. Appl. Phys. 64, 447 (1988).

${ }^{14}$ D. E. Aspnes and A. A. Studna, Phys. Rev. B 27, 985 (1983). 